\title{
Effects of visual reinforcer brightness and color on operant behavior of Siamese Fighting Fish'
}

\author{
JOHN G. GRABOWSKI AND TRAVIS THOMPSON $2, D E P A R T$ - \\ MENT OF PSYCHIATRY AND NEUROLOGY, UNIVERSITY \\ OF MINNESOTA, Minneapolis, Minnesota
}

The reinforcing effectiveness of models of Siamese Fighting Fish for operant responding of other Siamese Fighting Fish was found to vary as a function of the coloration of the model but not its brightness. Models colored unlike the $S$ were effective reinforcers whereas models colored similar to the $S$ 's coloration were relatively ineffective, confirming an earlier finding. The possible interaction between color and brightness remains unresolved.

A mirror or model of a male Siamese Fighting Fish (Betta splendens) presented to another Betta splendens can serve as an effective reinforcer for an operant response (Thompson, 1963). Increases in response rate associated with mirror presentation appear to be specific to the operant response rather than the result of a generalized activity increase (Goldstein, 1967; Hogan, 1967). The reinforcing effectiveness of a model of a male Betta splendens varies with the coloration of the model and the coloration of the $S$ (Thompson \& Sturm, 1965). Models whose coloration is unlike that of the $S$ appear to be most effective as reinforcers. The purpose of the present experiment was to determine whether the reinforcing effectiveness of a model of another male Betta splendens varies with its brightness as well as coloration.

Subjects. Two adult male Betta splendens of light red coloration were obtained from a local pet supplier.

Apparatus. The Ss were maintained in 2-liter Pyrex acquaria measuring 4.5 $\times 9 \times 10$ in., filled with conditioned tap water at $80^{\circ} \pm 1.0^{\circ}$. These aquaria served as test tanks when transferred to the response detection-reinforcement presentation apparatus. A $3 / 4 \times 1 \frac{1}{2} \times 1 \frac{1}{2}$ in. transparent lucite response channel was suspended in the tank, such that each transit of the fish through the channel interrupted a beam of light falling on photoconductors. By using external relay circuitry it was possible to detect only unidirectional passage through the channel, thus assuring that the $S$ was oriented toward the area of the tank wall where the reinforcing model was presented. The detachable model was suspended from a motor-driven chain which first presented the model frontally, then laterally along the front of the tank ( $10 \mathrm{in}$.). The total duration of exposure to the model was $11 \mathrm{sec}$, including a $4 \mathrm{sec}$ pause in the initial frontal approach. The apparatus has been described in greater detail by Sturm, Grabowski, \& Thompson (1966).

Nine structurally identical models of male Betta splendens were constructed for each S, varying only in color and brightness. The bodies of the models were plaster of paris casts of a male fish which had been sacrificed for making a rubber mold. Fins were copied from a photograph of a fish in maximal degree of midline fin erection during aggressive display, then cut from thin sheet steel (see Simpson, 1968 for a description of the display). The fins and two halves of the plaster of paris casts were cemented together, then painted. The eyes were black, pelvic fins and gill membranes bright red, and the remainder of the fish was one solid color. Since Betta splendens are most commonly red, blue or green, we made models in these three colors. For each color three brightnesses of paint were mixed and matched for reflected brightness using the Munsell Color Atlas. Thus, there were light, medium and dark degrees of brightness of red, blue and green colors, yielding nine combinations.

Procedure. The fish were maintained on a $14 \mathrm{~h}$ light-10 h dark cycle, with feeding approximately $1 \mathrm{~h}$ into the light period. One half hour after food had been placed in the tanks, the excess was siphoned out along with one-quarter of the water. At the end of each day this water was replaced with freshly conditioned tap water.

Two weeks were allowed for adapting to the foregoing routine, after which conditioning began. Experimental sessions were initiated $1 \mathrm{~h}$ following feeding by suspending the response channel in the tank and turning on a $15 \mathrm{~W}$ lamp along the rear wall of the aquarium. Each session lasted $\mathrm{l} h$ from the first response. During initial acquisition a model with complex markings unlike any that were used in subsequent testing was used. Baseline conditioning was continued for two weeks, when six day-to-day response rate variability had markedly diminished. During testing each session lasted for $1 \mathrm{~h}$ as before, and employed one of nine different models. Each block of nine sessions was replicated three times, thus requiring a total of 27 days to test each fish on three color-brightness series, as shown in Table 1. The order of brightness was varied systematically so each brightness of the three colors was presented in each order position once. The order of color presentation varied for the two Ss, but was constant for each $S$.

Results. Table 1 presents the number of responses per hour for each brightness-color combination over the 27 days. There is a gradual overall rate increase for all combinations over the 27 days. Since the baseline rate progressively increased, comparisons can best be made by expressing response rates as ratios during successive nine day blocks (i.e., responses Day $\mathrm{n} \div \Sigma$ responses Day 1...Day 9). Ratios of response rates were rank ordered and assigned ranks $(1=$ highest rate ratio and $27=$ lowest ratio $)$, yielding an ordinal measure of relative response rates as shown in Fig. 1. While relative response rate varied directly with brightness for the red and blue models there is an inverse relation between brightness and response rate with green models. Applying a Friedman two way analysis of variance to ranks by color, $\mathrm{X}^{2}=$ 8.7. The null hypothesis that these ranks were distributed normally by color was rejected $(\mathrm{df}=2, \mathrm{p}<.02)$. The differences in ranks by brightness failed to yield a significant difference.

Median tests of successive color pairs show that blue differed significantly from red $\left(\mathrm{X}^{2}=6.7, \mathrm{df}=2, \mathrm{p}<.05\right)$ and from green $\left(\mathrm{X}^{2}=7.2\right.$, df $\left.=2, \mathrm{p}<.05\right)$, but red and green did not differ significantly. Median tests of brightness pairs were not justified since the initial overall test failed to reject the null hypothesis of random distribution.

Discussion. The highest relative rates were maintained by the blue models, regardless of brightness, and the light red model maintained the lowest response rates. Since the Ss in this experiment were light red, these data are

Table 1

The order of presentation of Red (R), Blue (B) and Green (G) models of Light (L), Medium (M) and Dark (D) degrees of brightness over the $\mathbf{2 7}$ days of testing. The number of responses is recorded below the color-brightness designations for each day. Notice the gradual overall base rate increase over the course of testing.

Fish No. 4

\begin{tabular}{lccccccccc}
\multicolumn{1}{c}{ Day } & 1 & 2 & 3 & 4 & 5 & 6 & 7 & 8 & 9 \\
\hline Model & LR & MR & DR & LB & MB & DB & LG & MG & DG \\
No. of Rs & 22 & 30 & 30 & 42 & 25 & 36 & 25 & 30 & 24 \\
\hline \multicolumn{1}{c}{ Day } & 10 & 11 & 12 & 13 & 14 & 15 & 16 & 17 & 18 \\
\hline Model & MR & LR & DR & LB & MB & DB & LG & MG & DG \\
No. of Rs & 34 & 41 & 32 & 44 & 45 & 50 & 50 & 37 & 54 \\
\hline Day & 19 & 20 & 21 & 22 & 23 & 24 & 25 & 26 & 27 \\
\hline Model & DR & MR & LR & DB & LB & MB & MG & LG & DG \\
No. of Rs & 55 & 54 & 47 & 36 & 50 & 54 & 49 & 46 & 53 \\
\hline
\end{tabular}

Fish No. 2

\begin{tabular}{lccccccccc}
\multicolumn{1}{c}{ Day } & 1 & 2 & 3 & 4 & 5 & 6 & 7 & 8 & 9 \\
\hline Model & MB & LB & DB & MG & LG & DG & LR & MR & DR \\
No. of Rs & 23 & 29 & 34 & 37 & 26 & 16 & 11 & 41 & 37 \\
\hline \multicolumn{1}{c}{ Day } & 10 & 11 & 12 & 13 & 14 & 15 & 16 & 17 & 18 \\
\hline Model & DB & MB & LB & DG & LG & MG & DR & MR & LR \\
No. of Rs & 67 & 72 & 45 & 59 & 76 & 60 & 82 & 71 & 67 \\
\hline Day & 19 & 20 & 21 & 22 & 23 & 24 & 25 & 26 & 27 \\
\hline Model & LB & DB & MB & LG & DG & MG & MR & LR & DR \\
No. of Rs & 89 & 75 & 87 & 70 & 60 & 57 & 33 & 47 & 55 \\
\hline
\end{tabular}




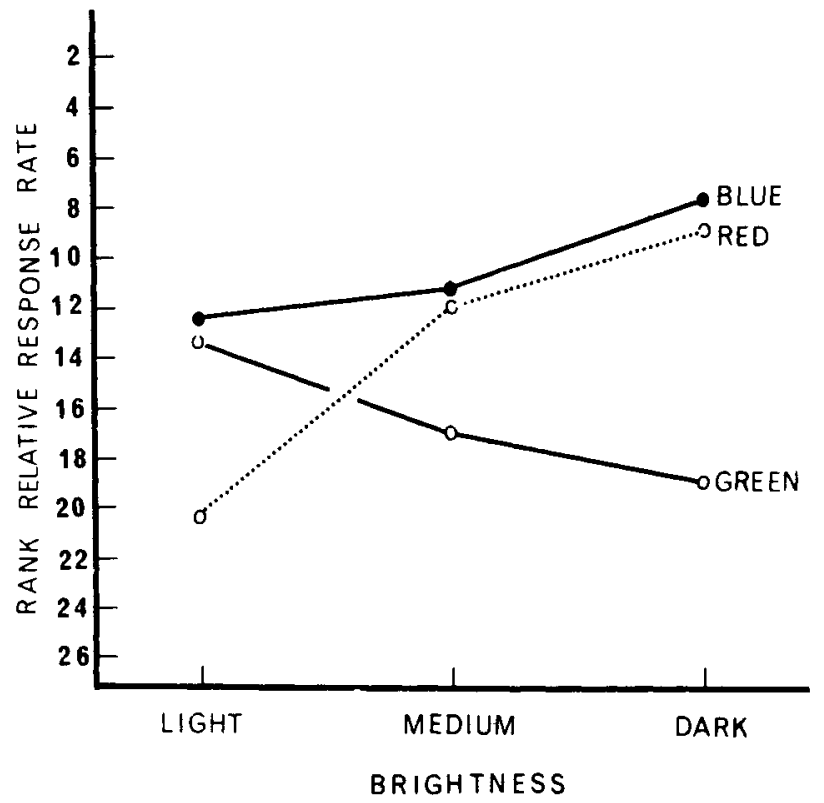

Fig. 1. The median ranks of relative response rates for each of the nine color-brightness combinations for both Ss. Each point is the median of six determinations, three for each $\mathbf{S}$.

consistent with earlier findings indicating that models whose color is like the $S$ are least effective as reinforcers and models unlike the $S$ are more effective (Thompson \& Sturm, 1965). The fact that all blue models were more effective than all other matched brightness combinations suggests that brightness is a relatively less significant factor determining reinforcing effectiveness. While differences in brightness failed to reach statistical significance this may be due to our inability to partial out interaction between brightness and color. ${ }^{3}$ The inverse relation between brightness and relative response rate in the green models contrasted with the direct relation for red and blue models certainly suggests such an interaction. This may also account for the failure to obtain a significant $X^{2}$ in the Green-Red median test despite the large differences in rank at dark and medium brightnesses.

The factors responsible for the differing curve for green models are not apparent. Similarity of the model coloration to that of water plants may account for some of the difference. However, aggressive display in the presence of predominantly green fish does not appear to be grossly different from that in the presence of red or blue fish (Simpson, in press), suggesting that other variables must be operating.

\section{REFERENCES}

GOLDSTEIN, S. R. Mirror image as, a reinforcer in Siamese Fighting Fish: A repetition with additional controls. Psychon. Sci, 1967, 7, 331-2.

HOGAN, J. A. Fighting and reinforcement in the Siamese Fighting Fish (Betta splendens). J. comp. physiol. Psychol. 1967, 64, 356-389.

SIMPSON, M. J. A. Threat display of the Siamese Fighting Fish. Anim. Behav., in press.

STURM. T., GRABOWSKI, J., \& THOMPSON, T. An apparatus for studying visual reinforcement in fish. Amer. J. Psychol., 1966, 79, 642-646.

THOMPSON, T. Visual reinforcement in Siamese Fighting Fish. Science, $1963,141,85-87$.

THOMPSON, T., \& STURM, T. J. exp. Anal. Behav., 1965, 5, 341-344. NOTES

1. This research was supported in part by National Science Foundation research grant GB-4518 to the University of Minnesota.

2. USPHS Special Postdoctoral Fellow 1-F3-MH-9011-01; Sub-Department of Animal Behaviour, University of Cambridge, England.

3. It was not possible to satisfy the assumptions of a parametric analysis of variance thereby necessitating the use of nonparametric statistics. Unfortunately, a nonparametric test for interaction is not available. 